Article

\title{
A Hybrid Electric Vehicle Dynamic Optimization Energy Management Strategy Based on a Compound-Structured Permanent-Magnet Motor
}

\author{
Qiwei $\mathrm{Xu}{ }^{1, *}$, Yunqi Mao ${ }^{1}$, Meng Zhao ${ }^{1}$ and Shumei Cui ${ }^{2}$ \\ 1 State Key Laboratory of Power Transmission Equipment \& System Security and New Technology, \\ Chongqing University, Chongqing 400044, China; myq2016@cqu.edu.cn (Y.M.); \\ zhaom2015@cqu.edu.cn (M.Z.) \\ 2 Department of Electrical Engineering, Harbin Institute of Technology, Harbin 150080, China; \\ cuism@hit.edu.cn \\ * Correspondence: xuqw@cqu.edu.cn; Tel.: +86-185-2327-8964
}

Received: 15 July 2018; Accepted: 21 August 2018; Published: 23 August 2018

\begin{abstract}
A dynamic optimization energy management strategy called Hybrid Electric Vehicle Based on Compound Structured Permanent-Magnet Motor (CSPM-HEV) is investigated in this paper. CSPM-HEV has obvious advantages in power density, heat dissipation efficiency, torque performance and energy transmission efficiency. This paper describes the topology and working principle of the CSPM-HEV, and analyzes its operating mode and corresponding energy flow laws. On this basis, the relationship about the power loss of the vehicle, the CSPM transmission ratio $i_{C S P M}$ and the CSPM-HEV power distribution coefficient $f_{1}$ were derived. According to the optimal combination of $\left(i_{C S P M}, f_{1}\right)$, the engine power and speed which minimize the power loss of the vehicle, were calculated, thus realizing the instantaneous optimal control of the vehicle. In addition, in order to improve the instantaneously optimized control processing speed, a neural network controller was established. The drive axle demand power, speed and battery State of Charge (SOC), were taken as input variables. Then, the engine power and speed were taken as output variables. The simulation results show that the average speed of the instantaneous optimization strategy after BP neural network optimization is increased by $98.1 \%$, the control effect is significant, and it has high application value.
\end{abstract}

Keywords: hybrid electric vehicle; compound structured permanent-magnet motor; energy management strategy; instantaneous optimization minimum power loss; back propagation (BP) neural network

\section{Introduction}

The compound structure permanent-magnet motor (CSPM) is a new electric transmission device. Its basic structure is shown in Figure 1. It consists of an inner rotor, an outer rotor and a stator. The outer rotor is composed of two layers of inner and outer layers. Ignoring the influence of magnetic field coupling, the motor can be regarded as a combination of two independent motors inside and outside. The internal motor EM1 is composed of an inner permanent magnet and an inner rotor, and the external motor EM2 is composed of an outer permanent magnet and a stator. The CSPM has two mechanical ports and two electrical ports, which realize efficient energy transmission, so the CSPM has broad application prospects in wind power generation, ship propulsion systems and hybrid electric vehicles [1]. 


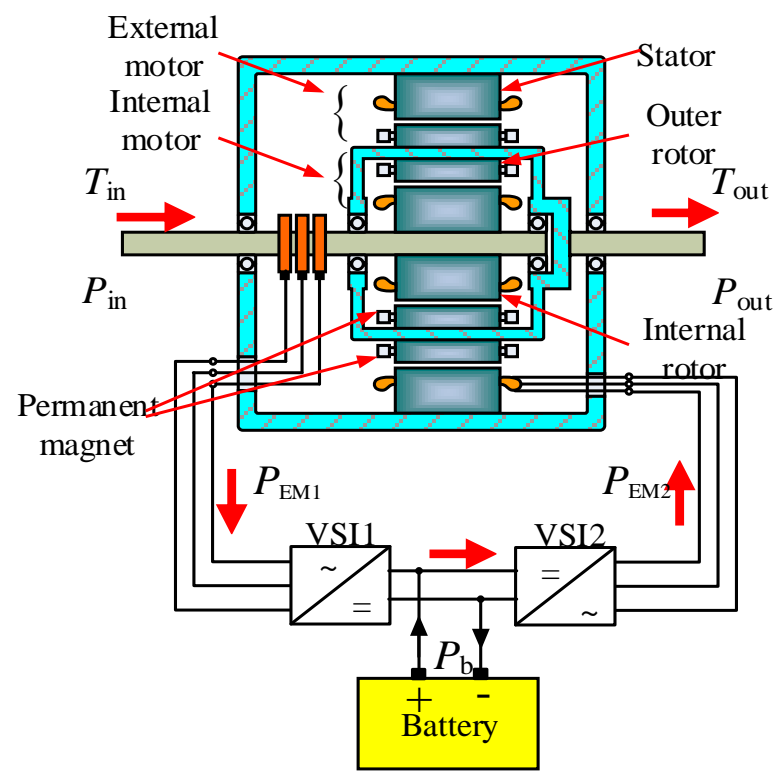

Figure 1. Structure of compound structure permanent-magnet motor.

A correlative research study demonstrates that a suitable Energy Management Strategy (EMS) can reduce HEV fuel consumption [2-19], and it is also beneficial to emissions reduction. In [2], dynamic programming was investigated for reducing the fuel consumption of vehicles when the future driving conditions are acquired. However, due to the computational complexity, the results obtained from dynamic programming cannot be executed directly. To solve this question, approximated dynamic programming [3] is proposed as an alternative solution. Since analytical optimization methods employ a mathematical equation to achieve the solution, their computation speed is faster than that of simple numerical methods. In this category, as an optimal control method, EMS based on Pontryagin's minimum principle (PMP) is proposed [4]. Unfortunately, this approach only works under the condition that the future driving conditions are known in advance. That is to say, vehicles must be able to communicate with road traffic systems [5]. In practice, currently this is difficult to achieve on a large scale.

For online implementation, in [6,7], the authors have developed a generic framework of online EMS for HEVs, where an evolutionary algorithm is used for online optimization of the power-split and battery SOC management. Due to the merit that the rule-based energy management algorithms are easy to implement in real time, this control strategy have been broadly applied in practical HEV EMS. In [8-10], rule-based EMS are used to split the power demand between the internal combustion engine (ICE) and the battery. In $[11,12]$ the authors adopted a rule-based EMS and they introduced a proper supervisory environment for a complex structure control. However, to create these rules, extensive engineering experience and extensive experimental data are needed. Moreover, this cannot improve the fuel economy significantly. To overcome this problem, $[13,14]$ propose a rule-based strategy combine with a fuzzy inference system. As a fuzzy system, due to its simple logic, it can be implemented in real time applications. In addition, it easy to model nonlinearity and uncertainty. However, the drawback of fuzzy systems is that they cannot achieve large modifications of the system modelling. This matter can be settled by the use of genetic algorithms (GAs) [15], which optimize the membership functions of the fuzzy controller for several matters while using appropriate fitness function. The trouble with GAs is random convergence of the solutions. Moreover, the operational speed of the optimization algorithm impedes the widespread application of GAs. To overcome this problem, the Artificial Neural Network (ANN) concept was introduced in [16-18]. ANNs are especially good at self-learning, adaptive ability and parallel distributed processing. Therefore, ANNs can be 
used to save the time consumed for optimization. They are suitable for improvement of instantaneous optimization strategies [19].

There are a variety of power source output couplings in HEVs with new topologies. The energy management strategy in traditional HEVs cannot achieve efficient power output distribution. Therefore, an optimized energy management strategy is proposed to improve the power, energy transmission efficiency and fuel economy of the new HEV. Through the optimized energy management strategy of this paper, the power, comfort, energy saving and environmental protection of HEVs are satisfied under different working conditions. The research of this paper promotes the development of electromagnetic continuously variable transmission. This paper develops a new theoretical foundation for the practical application of CSPM in HEVs.

Section 2 of this paper analyzes the operation mode of CSPM-HEV. Moreover, in order to facilitate the simulation analysis, the simulation model of CSPM-HEV was established and the simulation parameters of each component were determined in Section 3. In Section 4, based on the CSPM-HEV energy transfer characteristics, an instantaneous optimization energy management strategy based on a BP neural network was proposed, and the corresponding controller was designed in Section 5.

\section{CSPM-HEV Operating Mode Analysis}

After the structure optimization of CSPM, the magnetic field coupling, between internal and external motors in a CSPM can be neglected, so the CSPM can be seen as two independent permanent-magnet motors. Figure 2 is its equivalent schematic diagram. For simplification of the analysis, it was based on the equivalent permanent-magnet motor structure shown in Figure 2.
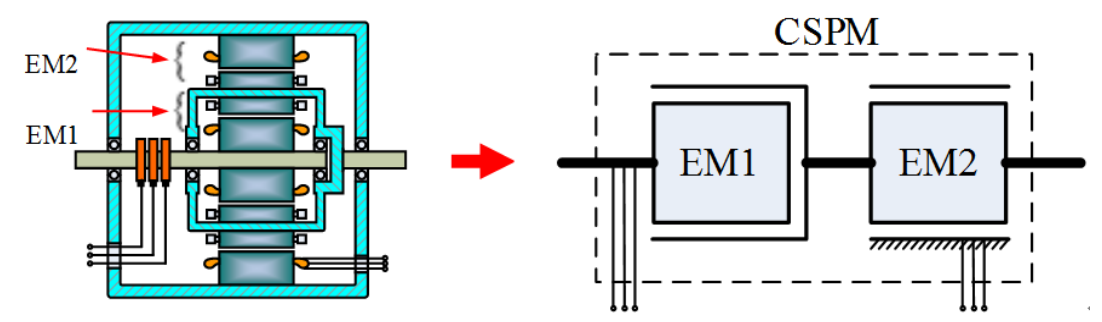

Figure 2. Equivalent diagram of compound-structure permanent-magnet motor.

According to the function of the CSPM in the HEV, the operating modes can be mainly divided into hybrid mode, starter mode, regenerative braking mode, generator mode and motor mode.

(1) Hybrid mode

When the vehicle demand torque is large or the battery SOC is low, the engine and the battery will jointly provide energy for vehicle. The CSPM works in the hybrid mode at this time, and its power flow is shown in Figure 3.

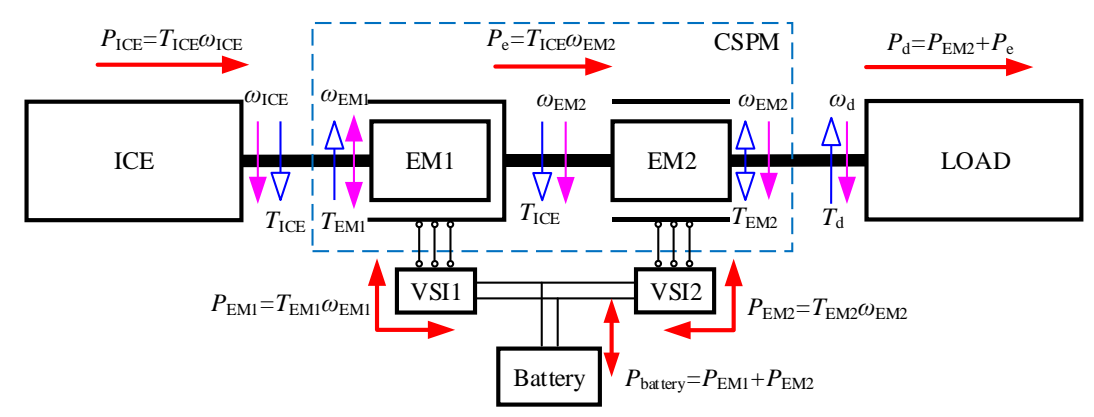

Figure 3. Hybrid mode and the related power flow diagram. 
(2) Starter mode

In a conventional vehicle, the engine needs an external force to start. This process is completed by an electric motor. However, the CSPM-HEV avoids this process, and the inner motor EM1 can realize this function. Its power flow is shown in Figure 4.

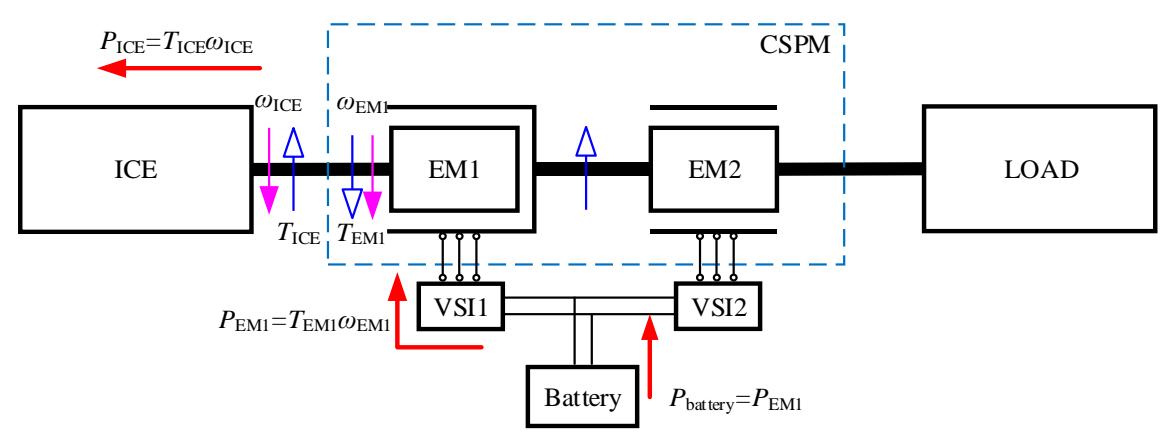

Figure 4. Starter mode and the related power flow diagram.

\section{(3) Generator mode}

If a vehicle is static for a short time and the battery SOC is low, the external motor of the CSPM stops working, and the engine drives the inner motor to operate as generator. Therefore, the battery is charged. Its power flow is shown in Figure 5.

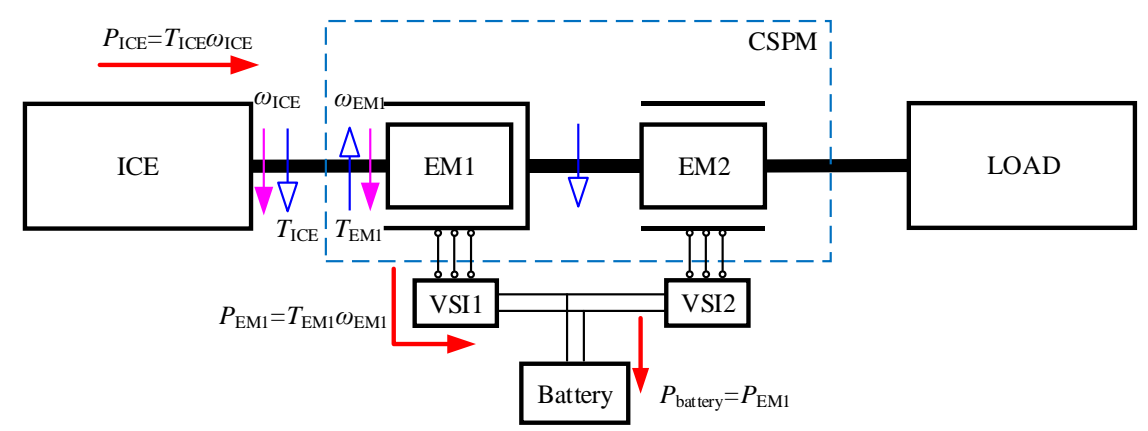

Figure 5. Generating mode and the related power flow diagram.

(4) Motor mode

When the vehicle runs at low speed and the battery SOC is high, the CSPM-HEV works in the pure electric mode. At this time, the inner motor EM1 and the engine do not work, and the outer motor EM2 works as a motor to directly drive the vehicle, and the system energy is all provided by the battery. Its power flow is shown in Figure 6.

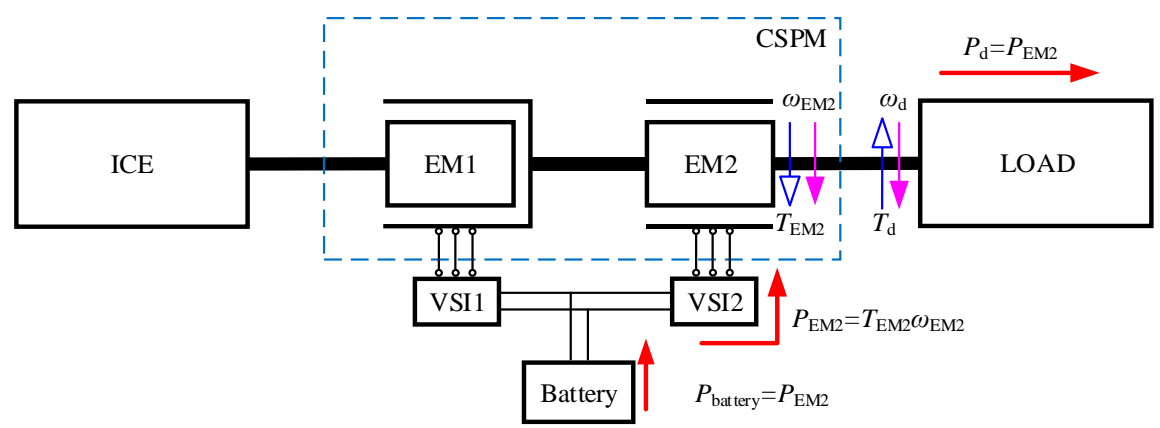

Figure 6. Motor mode and the related power flow diagram. 
(5) Regenerative braking mode

When the vehicle brakes, the EM2 works in the power generation state, and the recovered energy is stored in the battery. The power flow is shown in Figure 7.

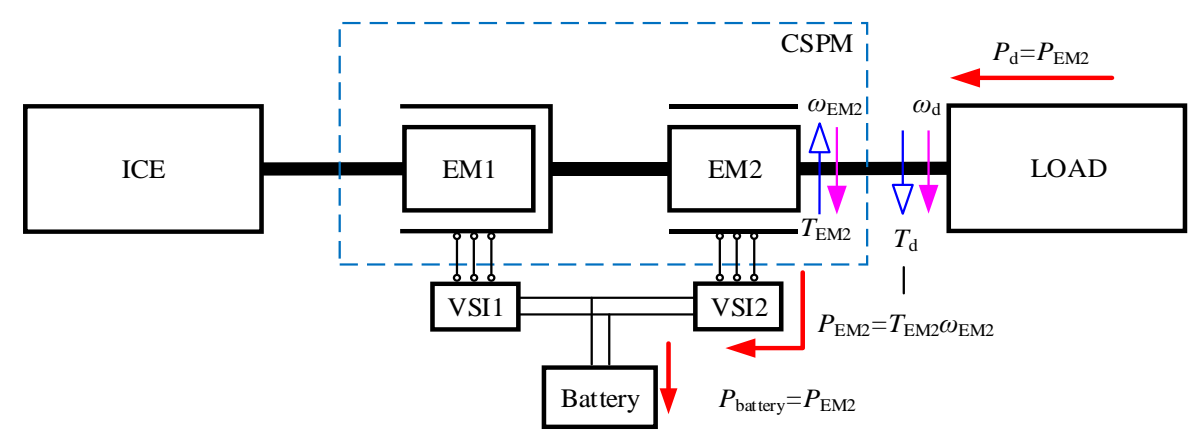

Figure 7. Regenerative braking mode and the related power flow diagram.

\section{CSPM-HEV Model and Parameters}

According to the mathematical model of the ICE, CSPM, battery and other components, the simulation models were built in the MATLAB/Simulink (MATLAB 2014a, MathWorks, Natick, MA, USA) environment [20,21]. Afterwards, due to the connection relationship of each component, a vehicle model of the CSPM was established based on the ADVISOR (ADVISOR 2002, AVL List GmbH, Graz, Austria) simulation platform. The entire model is shown in Figure 8. Simulation parameters and main component parameters of CSPM-HEV are shown in Tables 1 and 2 respectively.
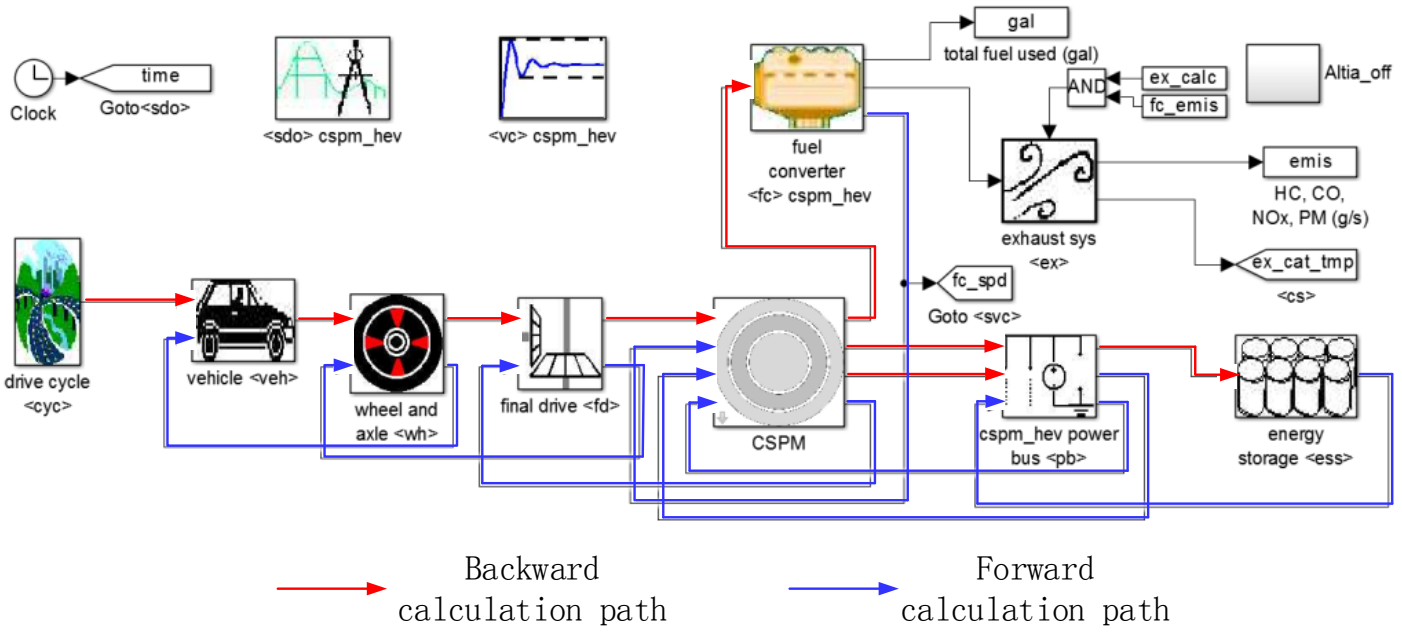

Figure 8. Simulation model of CSPM-HEV.

Table 1. Simulation parameters of CSPM-HEV.

\begin{tabular}{cccc}
\hline Parameter & Data & Parameter & Data \\
\hline Curb weight & $1360 \mathrm{~kg}$ & Main reducer efficiency & 0.95 \\
Frontal area & $1.746 \mathrm{~m}^{2}$ & Rolling resistance coefficient & 0.01 \\
Air resistance coefficient & 0.3 & Tire Rolling radius & $0.2928 \mathrm{~m}$ \\
Final drive ratios & 3.905 & & \\
\hline
\end{tabular}


Table 2. The main component parameters of CSPM-HEV.

\begin{tabular}{ccc}
\hline Component & Parameter & Data \\
\hline \multirow{2}{*}{ ICE } & Power & $43 \mathrm{~kW}$ \\
& Torque & $100 \mathrm{Nm}$ \\
& Type & Double magnet type \\
& External motor power & $30 \mathrm{~kW}$ \\
CSPM & Internal motor power & $20 \mathrm{~kW}$ \\
& Torque peak of external motor & $305 \mathrm{Nm}$ \\
& Torque peak of internal motor & $100 \mathrm{Nm}$ \\
Battery & Rated voltage & $300 \mathrm{~V}$ \\
\hline
\end{tabular}

\section{Instantaneous Energy Management Strategy Based on BP Neural Network}

To some extent, the instantaneous optimization energy management strategy (IO-EMS) compensates for the shortcomings of energy management strategies based on rules and global optimization. Due to the independence from expert experience, the optimized control can be achieved at each moment. Compared with global optimization, IO-EMS can adapt to different working conditions. However, due to the massive calculations required, IO-EMS cannot guarantee the real-time performance, which hinders its application.

Artificial Neural Networks (ANNs) are a computing system formed by connecting a number of simple processing units (neurons). It is also called "neural network", which mimics the operating mechanism of the human brain and enables the machine to have some features of the human brain. The control system utilized the continuous learning, profound memory and adaptability of the neural network, so that the control system can realize the non-linear mapping between input and output. Neural networks are suitable for modeling and the control of systems with many uncertainties [22].

This section develops an IO-EMS based on the principle of "minimum power loss", and it proposes a real-time energy management strategy based on a BP neural network (BP-EMS). BP-EMS improves the poor real-time performance of IO-EMS. The optimal solution at each moment in each operating condition was collected as a training sample of the neural network. Then, the BP neural network was trained. Afterwards, the BP neural network controller was built.

The optimization based on the "minimum power loss" principle is to find a set of values, which minimizes power loss among all the power flow combinations, and these values are used to calculate the torque and speed of engine demand. Thus, the minimum power loss of the system is achieved, and the optimal vehicle efficiency is achieved eventually.

The speed difference between the two shafts is continuously variable, which is similar to the traditional continuously variable transmission, so that the transmission ratio $i_{C S P M}$ can be defined according to the concept in the mechanical transmission:

$$
i_{C S P M}=\frac{\omega_{I C E \_G}}{\omega_{E M 2}}
$$

where $\omega_{I C E_{-} G}$ is output speed of the engine after the gear.

When the CSPM-HEV runs in hybrid mode, the power between the EM1 and the engine has the following relationship:

$$
P_{E M 1}=T_{E M 1}\left(\omega_{E M 2}-\omega_{I C E_{-} G}\right)=P_{I C E}\left(1-\frac{1}{i_{C S P M}}\right)
$$

From Equation (2), we can get that if $i_{C S P M}>1$, EM1 works in the power generation state; if $i_{C S P M}<1$, EM1 works in the electric state.

When the CSPM-HEV runs, the required power of the drive axle is definite. For simplification of power flow analysis, the engine and the EM1 can be regarded as a whole, and they are defined as the engine block, as shown in Figure 9. 


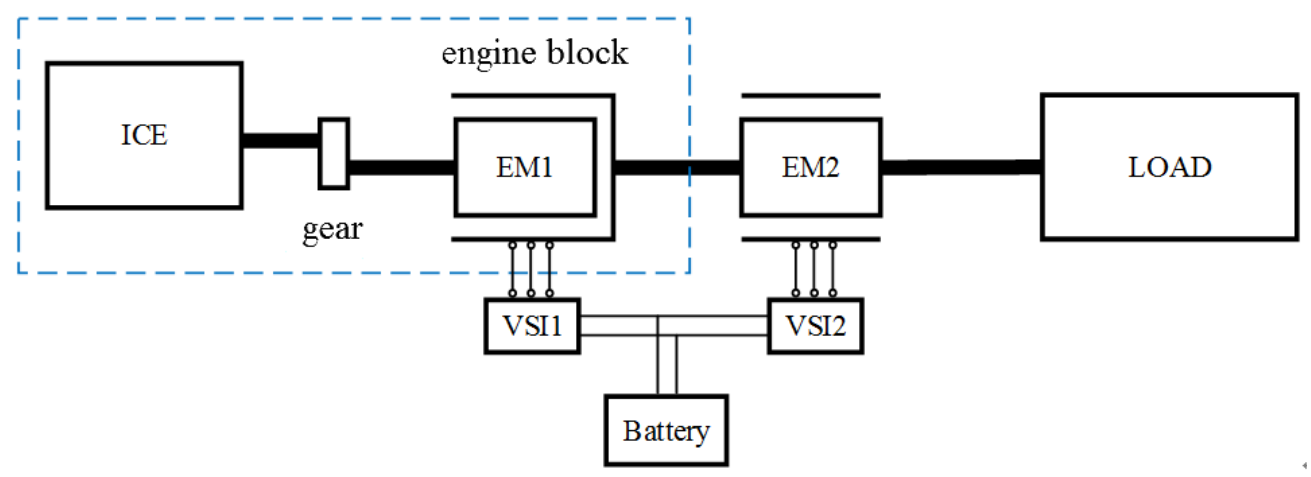

Figure 9. ICE group block diagram.

Since the external motor EM2 is independent with the engine, the power distribution coefficient $f_{1}$ can be defined. At this time, the output power of the engine group and the EM2 are:

$$
\begin{gathered}
P_{I C E}+P_{E M 1}=f_{1} \times P_{\text {req }} \\
P_{E M 2}=\left(1-f_{1}\right) \times P_{r e q}
\end{gathered}
$$

In the equation, $P_{r e q}$ is the required power of the axle.

Combining Equation (2) with Equation (3), the power of the engine and internal motor EM1 can be expressed as:

$$
\begin{gathered}
P_{I C E}=\frac{i_{C S P M}}{2 i_{C S P M}-1} \times f_{1} \times P_{\text {req }} \\
P_{E M 1}=\frac{i_{C S P M}-1}{2 i_{C S P M}-1} \times f_{1} \times P_{\text {req }}
\end{gathered}
$$

Due to the inevitable power loss during the powertrain transmission and the mechanical transmission, the power loss of the drive axle is mainly concentrated in four parts: engine, internal motor EM1 and its controller, external motor EM2 and its controller, battery, respectively. The total system power loss $P_{\text {loss_real }}$ can be expressed as:

$$
\begin{gathered}
P_{\text {loss_real }}=P_{I C E} \times \frac{1-\eta_{I C E}}{\eta_{I C E}}+\left|P_{E M 1}\right| \times \frac{1-\eta_{E M 1}}{\eta_{E M 1}} \\
+\left|P_{E M 2}\right| \times \frac{1-\eta_{E M 2}}{\eta_{E M 2}}+\left|P_{b a t}\right| \times \frac{1-\eta_{\text {bat }}}{\eta_{\text {bat }}} \\
P_{\text {bat }}=P_{E M 1} \times \frac{1}{\eta_{E M 1}}+P_{E M 2} \times \frac{1}{\eta_{E M 2}}
\end{gathered}
$$

In the above equations, $\eta_{I C E}$ is the engine efficiency; $\eta_{E M 1}$ is the EM1 and its controller efficiency; $\eta_{E M 2}$ is the EM2 and its controller efficiency; and $\eta_{b a t}$ is the battery efficiency. They have the following relationship:

$$
\left\{\begin{array}{l}
0 \leq \eta_{I C E} \leq 1 \\
0 \leq \eta_{E M 1} \leq 1 \\
0 \leq \eta_{E M 2} \leq 1 \\
0 \leq \eta_{\text {bat }} \leq 1
\end{array}\right.
$$

Substituting the output power of engine and EM1/EM2 into Equation (7): 


$$
\begin{aligned}
& P_{\text {loss_real }}=P_{\text {req }} \times\left[\frac{i_{C S P M}}{2 i_{C S P M}-1} \times f_{1} \times \frac{1-\eta_{I C E}}{\eta_{I C E}}\right. \\
& +\left|\frac{i_{C S P M}-1}{2 i_{C S P M}-1}\right| \times f_{1} \times \frac{1-\eta_{E M 1}}{\eta_{E M 1}}+\left|1-f_{1}\right| \times \frac{1-\eta_{E M 2}}{\eta_{E M 2}} \\
& \left.+\left|\left(1-f_{1}\right) \times \frac{1}{\eta_{E M 2}}+\frac{i_{C S P M}-1}{2 i_{C S P M}-1} \times f_{1} \times \frac{1}{\eta_{E M 1}}\right| \times \frac{1-\eta_{\text {bat }}}{\eta_{\text {bat }}}\right]
\end{aligned}
$$

In the CSPM-HEV, the energy in the battery comes from two parts: one is the energy recovered by regenerative braking, and the other is converted from the mechanical energy of the engine. The latter accounts for a large proportion. Since the battery will wear out during charging and discharging, when analyzing the system loss, the loss of the battery should be considered. Therefore, the battery loss compensation factor is introduced, which can be expressed as:

$$
K=\frac{1}{\bar{\eta}_{I C E} \times \bar{\eta}_{C S P M} \times \bar{\eta}_{b a t}}-1
$$

In the equation, $\bar{\eta}_{I C E}, \bar{\eta}_{C S P M}, \bar{\eta}_{b a t}$ represent the average efficiency of the engine, CSPM, and battery, respectively.

When the battery is discharged, the system power loss can be expressed as:

$$
\begin{aligned}
& P_{\text {loss }}=P_{\text {req }} \times\left[\frac{i_{C S P M}}{2 i_{C S P M}-1} \times f_{1} \times \frac{1-\eta_{I C E}}{\eta_{I C E}}\right. \\
& +\left|\frac{i_{C S P M}-1}{2 i_{C S P M}-1}\right| \times f_{1} \times \frac{1-\eta_{E M 1}}{\eta_{E M 1}}+\left|1-f_{1}\right| \times \frac{1-\eta_{E M 2}}{\eta_{E M 2}} \\
& \left.+\left|\left(1-f_{1}\right) \times \frac{1}{\eta_{E M 2}}+\frac{i_{C S P M}-1}{2 i_{C S P M}-1} \times f_{1} \times \frac{1}{\eta_{E M 1}}\right| \times \frac{1-\eta_{\text {bat }}+K}{\eta_{\text {bat }}}\right]
\end{aligned}
$$

When the battery is charging, the system power loss $P_{\text {loss }}$ is equal to $P_{\text {loss_real }}$ :

$$
P_{\text {loss }}=P_{\text {loss_real }}
$$

From Equation (10), it can be seen that the efficiency of each system component can be obtained by looking it up in the table, according to the corresponding $i_{C S P M}$ and $f_{1}$. Consequently, the power loss of the vehicle will be only related to these two variables. Therefore, when the power distribution coefficient $f_{1}$ is a definite value, and Equation (10) is a linear equation with one unknown. It can be known from MATLAB calculation that the power loss becomes small firstly and then becomes large as the gear ratio $i_{C S P M}$ increases. In this process, there is a minimum value of the power loss. If within the value range of the power distribution coefficient $f_{1}$, all minimum values form a set, then, the minimum value in this set is the minimum power loss under the specific operating condition. Similarly, if the transmission ratio is determined first, then the relationship between the power distribution coefficient and the power loss is same as the above conclusion.

At each moment under the IO-EMS, there are multiple combinations of $\left(i_{C S P M}, f_{1}\right)$, and the power loss of each combination will be compared with each other, to find the combination that minimize the power loss:

$$
J^{*}=\min \left\{P_{\text {loss }}\left(i_{C S P M}, f_{1}\right)\right\}
$$

According to the $\left(i_{C S P M}, f_{1}\right)$ combination at each moment, the required power, speed and torque of the engine, EM1 and EM2 can be calculated under the current combination:

$$
\left\{\begin{array}{l}
P_{I C E}=\frac{i_{C S P M}}{2 i_{C S P M}-1} \times f_{1} \times P_{\text {req }} \\
\omega_{I C E}=\frac{i_{C S P M} \times \omega_{E M 2}}{i_{\text {gear }}} \\
T_{I C E}=\frac{P_{I C E}}{\omega_{I C E}}
\end{array}\right.
$$




$$
\begin{gathered}
\left\{\begin{array}{l}
\omega_{E M 1}=\left(i_{C S P M}-1\right) \times \omega_{E M 2} \\
T_{E M 1}=-T_{I C E}
\end{array}\right. \\
\left\{\begin{array}{l}
T_{E M 2}=\frac{\left(1-f_{1}\right) \times P_{\text {req }}}{\omega_{E M 2}} \\
\omega_{E M 2}=\omega_{\text {req }}
\end{array}\right.
\end{gathered}
$$

In the equation, $i_{\text {gear }}$ is the transmission ratio of the gear, $\omega_{\text {req }}$ is the required speed of the axle.

In the process of solving the IO-EMS algorithm, the power, speed, and torque of the engine, EM1, and EM2 must satisfy the following constraints:

$$
\left\{\begin{array}{l}
T_{I C E \_m i n} \leq T_{I C E} \leq T_{I C E \_ \text {max }} \\
\omega_{I C E \_\min } \leq \omega_{I C E} \leq \omega_{I C E \_\max } \\
P_{I C E \_ \text {min }} \leq P_{I C E} \leq P_{I C E \_\max } \\
T_{E M 1,2 \_\min } \leq T_{E M 1,2} \leq T_{E M 1,2 \_\max } \\
\omega_{E M 1,2 \_\min } \leq \omega_{E M 1,2} \leq \omega_{E M 1,2 \_\max } \\
P_{E M 1,2 \_\min } \leq P_{E M 1,2} \leq P_{E M 1,2 \_\max } \\
S O C_{\min } \leq S O C \leq S O C_{\max }
\end{array}\right.
$$

The implementation of the IO-EMS based on the minimum power loss is as follows: At every moment when the CSPM-HEV operates in the hybrid mode, all possible combinations $\left(i_{C S P M}, f_{1}\right)$ are listed firstly, then, these combinations are adopted to calculate the power, torque, and speed required for the engine, EM1, and EM2 by Equations (15)-(17). The calculation results must satisfy the constraints shown in Equation (18). Those combinations that do not meet the constraints will be discarded. Afterwards, according to the required power, torque and speed of the engine, EM1 and EM2, the efficiency of the component is obtained by looking up the table. After this, the power loss, which satisfy the condition, is calculated by Equations (12) and (13). When the loss $P_{\text {loss }}$ is minimum, the corresponding $\left(i_{C S P M}, f_{1}\right)$ combination is obtained. Next, according to Equations (15)-(17), the power, speed, and torque of the engine, EM1, and EM2 corresponding to the combination are calculated. Eventually, these data are used to control the system operation, and the minimum vehicle power loss is realized.

\section{Neural Network Controller Design}

In order to realize the Back-Propagation Neural Network Energy Management Strategy (BP-EMS), this paper designed a neural network controller, structure of which is shown in the Figure 10. According to the three input variables in the IO-EMS, three neurons were designed on the input layer, they are axle power demand, axle speed demand and battery SOC, respectively. Afterwards, two neurons were designed on the output layer, these two neurons represented the engine power and speed respectively. By the engine speed and torque, the operating point of the internal and external motors can be calculated, so that realizing the efficient energy management of the system.

For the three-layer BP neural network, the number of input and output layer neurons is determined, so the hidden layer neurons determine the network structure and have an important impact on network performance. If the number of neurons in the hidden layer is too small, the network is difficult to train and its performance will be poor; if there are too many hidden layer neurons, the training time of the network will be increased greatly, and the training will easily fall into a local minimum without the optimal advantage. Moreover, the excessive neurons will also bring difficulties for hardware and software implementations. According to the principle of simplifying the network structure as much as possible, this paper determined the number of hidden layer neurons by continuous experimentation. 


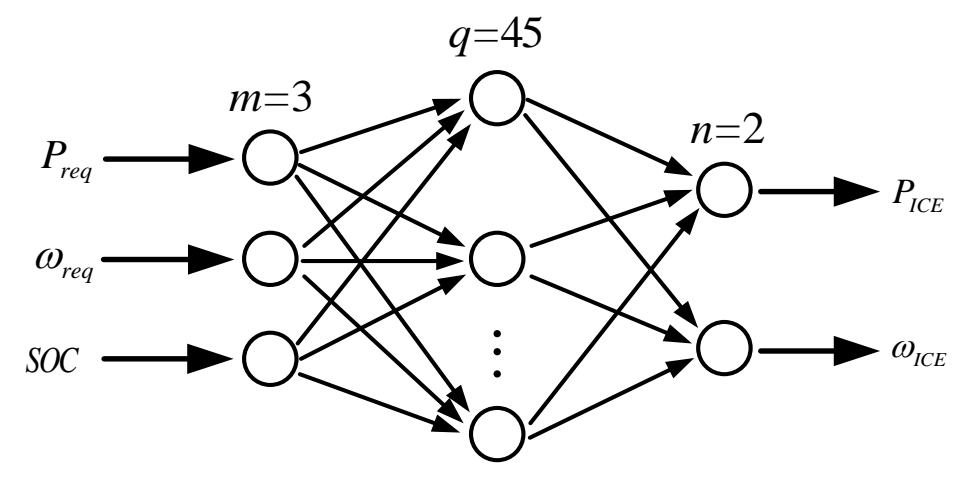

Figure 10. BP neural network energy management controller structure diagram.

\subsection{BP Neural Network Training}

There is an important relationship between the selection of training samples and their performance in neural networks, so the following aspects should be noted when learning the sample selection of neural network controllers:

(1) The sample should be widely representative and reflect the working characteristics of all possible operating conditions of the HEV.

(2) The neural network after the sample learning has good generalization ability.

(3) Do not have too many samples, otherwise it may lead to over-fit of the network.

In the BP neural network controller of this paper, the input and output variables such as vehicle power, speed, and battery SOC vary greatly in magnitude, and they need to be normalized before training, that is, all the data were converted into $(0,1)$, to eliminate the impact of different orders of magnitude on the network. The linear function method was used here, as shown in the following equation:

$$
\overline{x_{i}}=\frac{x_{i}-x_{\min }}{x_{\max }-x_{\min }}
$$

In the equation, $x_{i}$ represents the sample; $x_{\max }$ and $x_{\min }$ represent the sample maximum and minimum values respectively; $\overline{x_{i}}$ is the standardized sample.

In order to overcome the drawbacks of the standard BP algorithm, such as the difficulty in adjusting initial network weight, learning rate and momentum coefficient, and the long training time and slow convergence rate, the neural network controller adopted Levenberg-Marquardt algorithm for sample learning. The flow chart is shown in the Figure 11, and the training steps are as follows:

Step 1: The required power, speed, and battery SOC are putted in the training sample to the network and the error between the output and the target value $e_{i}(W)\left(i=1, \ldots, N_{1}, N_{1}\right.$ as the total number of training samples) is calculated. Here, the error indicator was defined:

$$
E_{k}(W)=\frac{1}{2} \sum_{i=1}^{N_{1}} e_{i}^{2}(W)
$$

In the equation, $W$ is the vector group formed by the weights and thresholds in the network, $k$ is the number of iterations of network learning.

Step 2: If the requirements are satisfied. If yes, training will be saved and ended. If not, go to the next step. 
Step 3: The Jacobian matrix is calculated:

$$
J(W)=\left[\begin{array}{cccc}
\frac{\partial e_{1}(W)}{\partial W_{1}} & \frac{\partial e_{1}(W)}{\partial W_{2}} & \cdots & \frac{\partial e_{1}(W)}{\partial W_{n}} \\
\frac{\partial e_{2}(W)}{\partial W_{1}} & \frac{\partial e_{2}(W)}{\partial W_{2}} & \cdots & \frac{\partial e_{2}(W)}{\partial W_{n}} \\
\vdots & \vdots & \ddots & \vdots \\
\frac{\partial e_{N_{1}}(W)}{\partial W_{1}} & \frac{\partial e_{N_{1}}(W)}{\partial W_{2}} & \cdots & \frac{\partial e_{N_{1}}(W)}{\partial W_{n}}
\end{array}\right]
$$

Step 4: The adjustment rate is solved through the following equation:

$$
\Delta W=-\left[\mu I+J(W)^{\mathrm{T}} J(W)\right]^{-1} J(W)^{\mathrm{T}} e(W)
$$

In the equation, $\mu$ is a non-negative number that indicates the speed of network learning.

$$
e(W)=\left[e_{1}(W), e_{2}(W), \ldots, e_{N_{1}}(W)\right]^{\mathrm{T}}
$$

Step 5: $W+\Delta W$ is calculated by Equation (20). If $E_{k+1}(W)<E_{k}(W)$, then $\mu=\mu / 2$, $W_{k+1}=W_{k}+\Delta W$, skip to Step 1; otherwise, go to Step 4 .

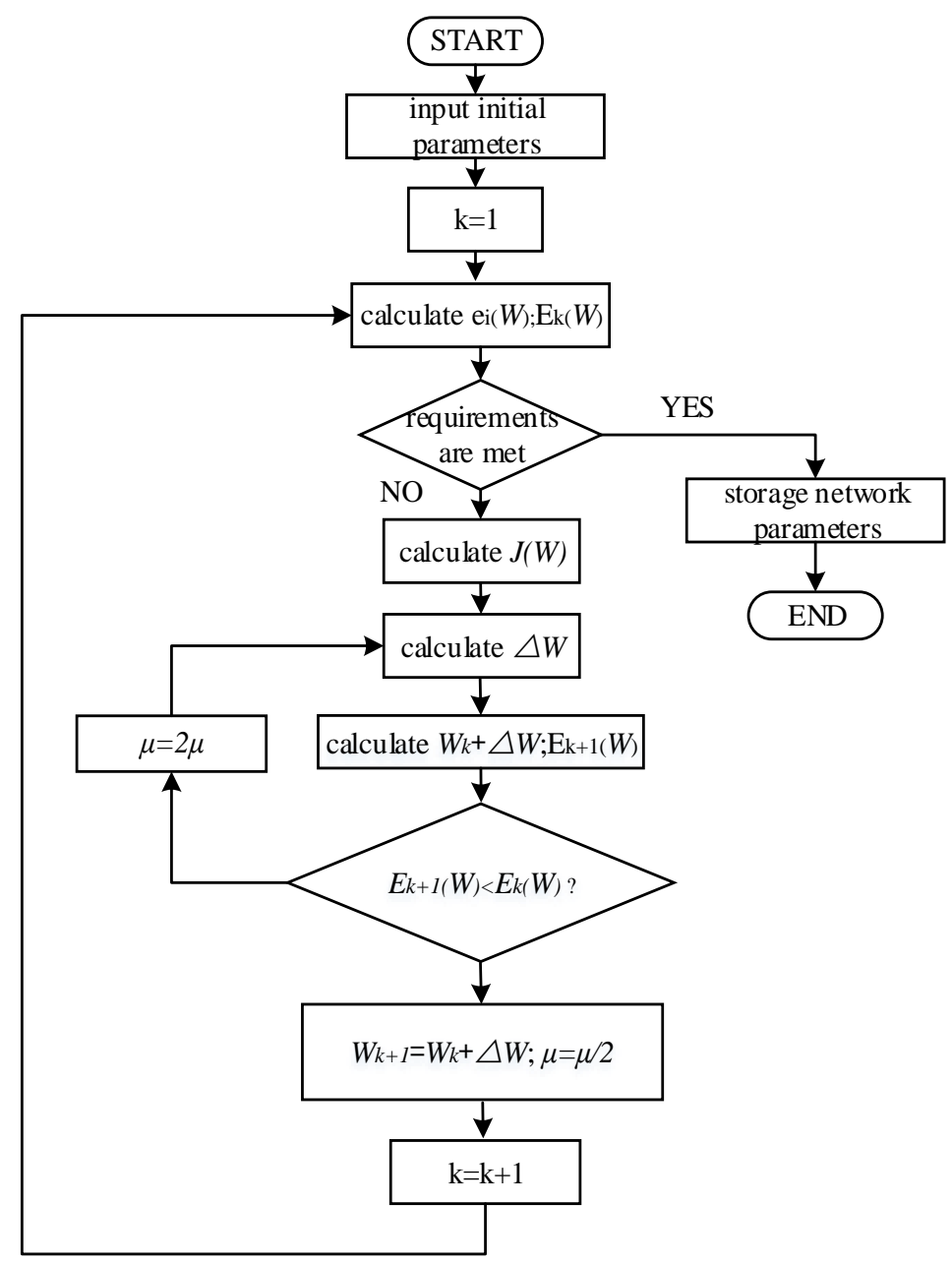

Figure 11. Levenberg-Marquardt algorithm flowchart. 


\subsection{Analysis of Simulation Results}

In order to verify the control effect of the BP-EMS, the Urban Dynamometer Driving Schedule (UDDS), US06 Highway Cycle (US06 HWY), and New European Driving Cycle (NEDC) were simulated and the results were compared with the corresponding results of IO-EMS. Figure 12 shows the engine output power and torque command value based on the two control strategies under UDDS conditions. It can be seen from this figure, if engine power and torque are negative values, the invalid command values of the engine in the closed state are removed, the BP neural network controller will give a good effect on the sample learning. It realizes the nonlinear mapping between vehicle demand power and speed, battery SOC and engine power and torque command value.

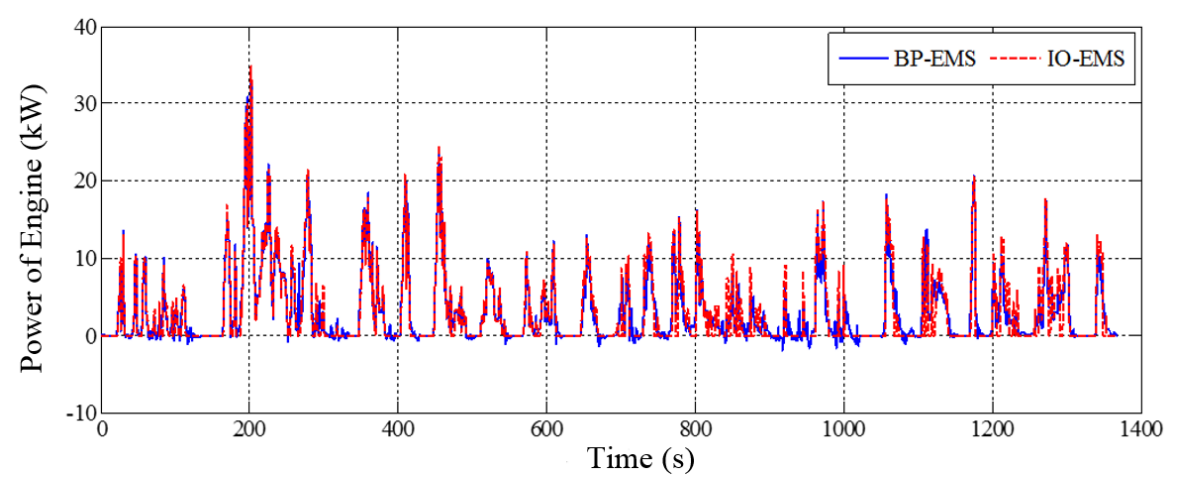

(a)

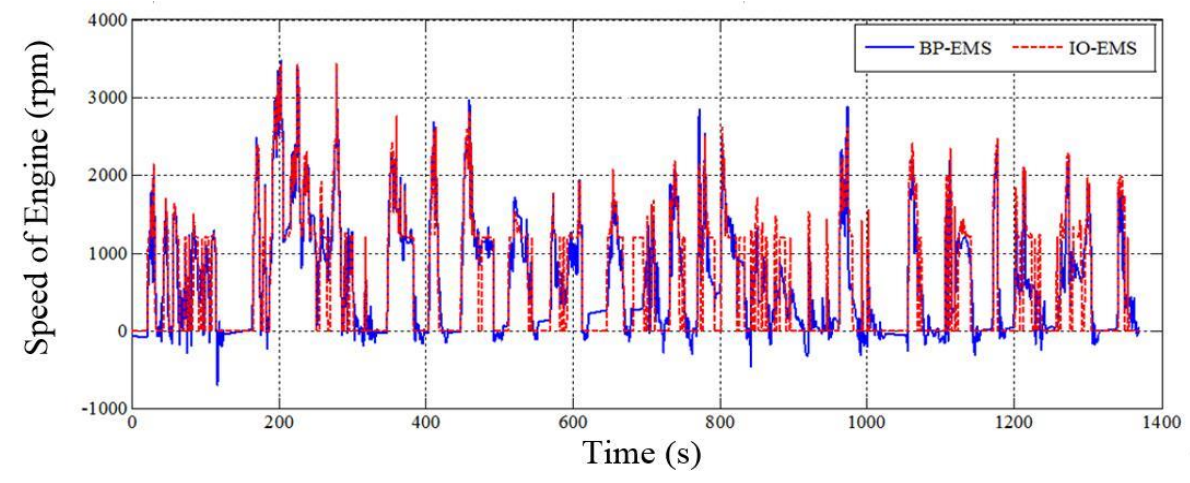

(b)

Figure 12. Power (a) and speed (b) of ICE during UDDS driving cycle.

From simulation results of US06 HWY and NEDC driving cycle, it can get the same analysis results as UDDS driving cycle. Simulation results of US06 HWY and NEDC are shown in Figures 13 and 14 respectively. 


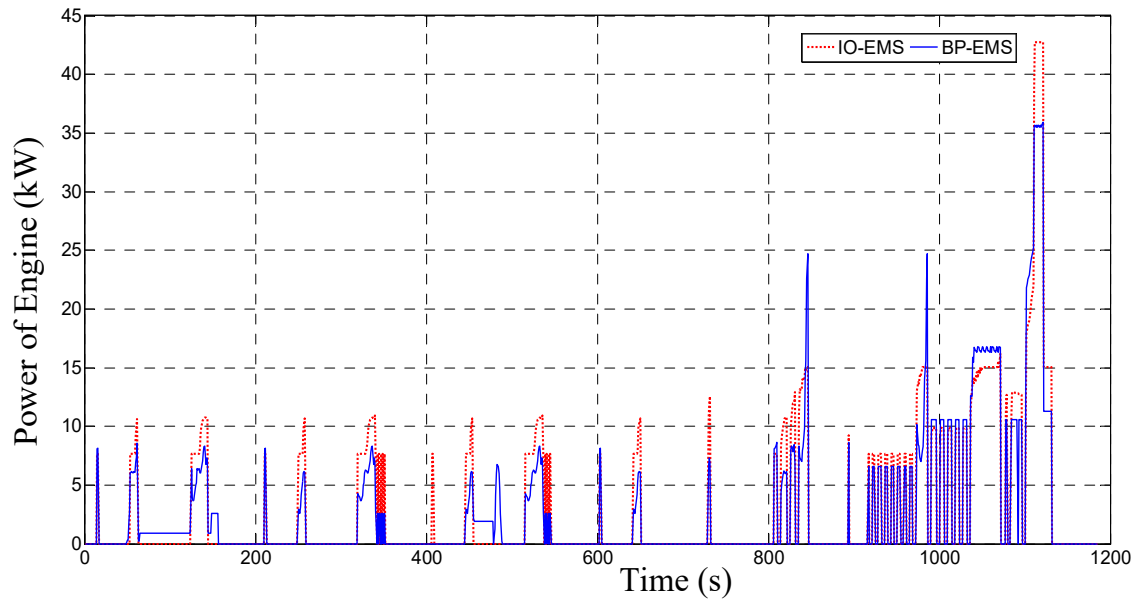

(a)

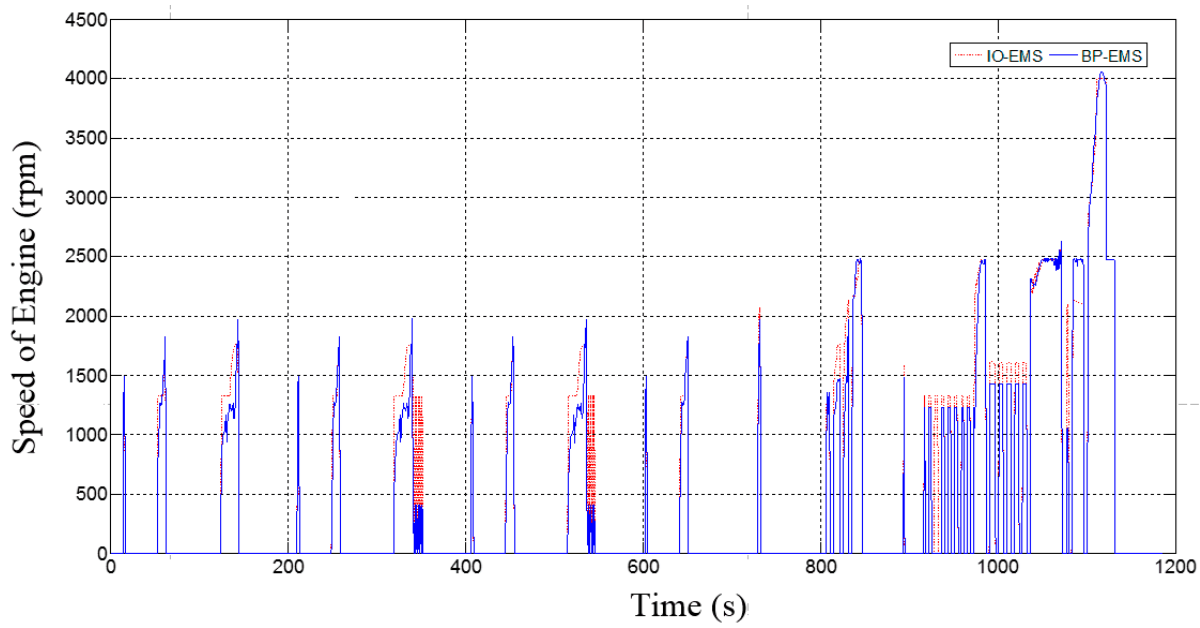

(b)

Figure 13. Power (a) and speed (b) of ICE during NEDC driving cycle.

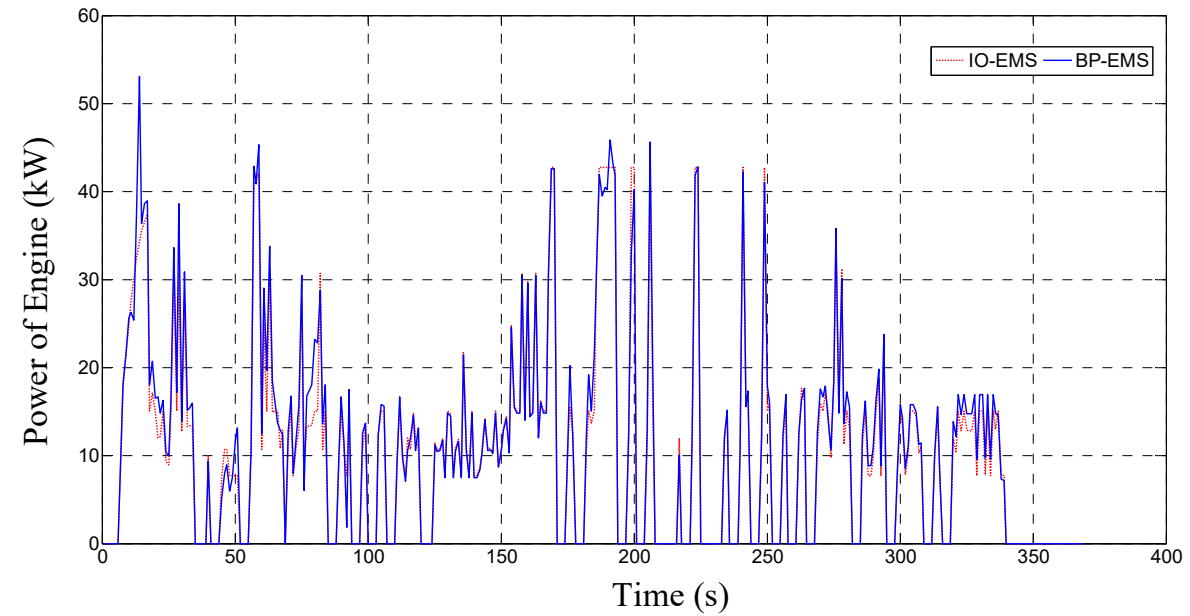

(a)

Figure 14. Cont. 


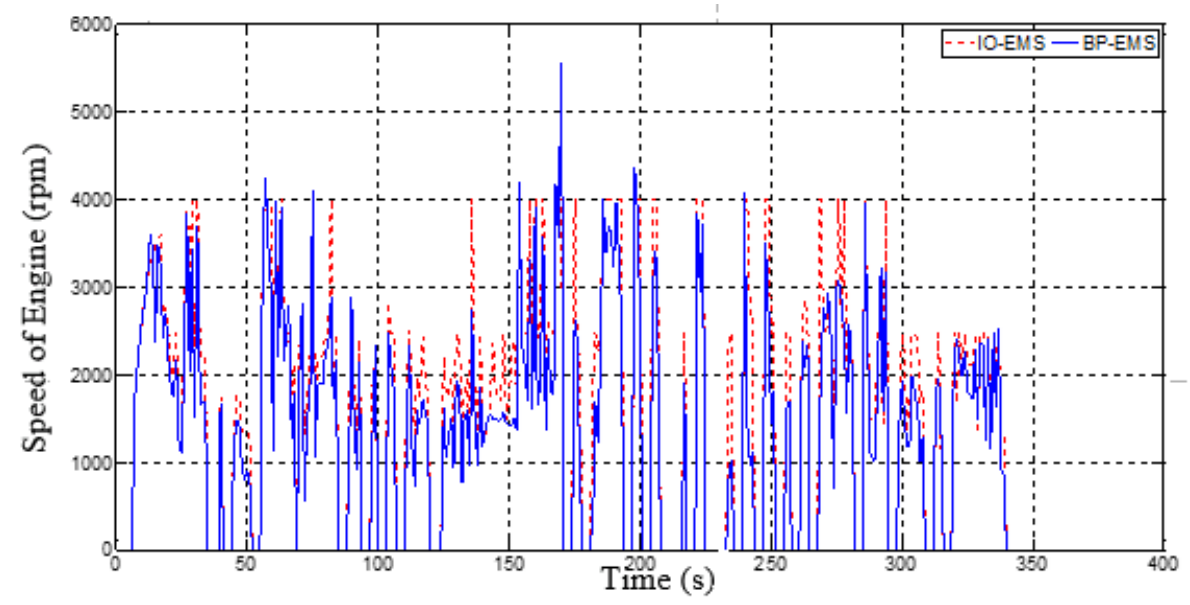

(b)

Figure 14. Power (a) and speed (b) of ICE during US06 driving cycle.

In order to make the simulation results more convincing, this paper uses Mean Square Error (MSE) to process the data obtained from the two control strategies in different working conditions. The results are shown in the Table 3. For the simplification of calculation, the unit of speed is a thousand revolutions per minute. The calculation results show that the control effect of the two control strategies is similar, and the introduction of neural network does not decrease the control performance of instantaneous optimization.

Table 3. Mean square error of obtained data from two control strategies.

\begin{tabular}{ccc}
\hline Cycle Condition & Parameter & Mean Square Error \\
\hline \multirow{2}{*}{ UDDS } & Power of Engine & 3.6 \\
& Speed of Engine & 0.063 \\
US06 & Power of Engine & 5.5 \\
& Speed of Engine & 0.135 \\
\multirow{2}{*}{ NEDC } & Power of Engine & 9.8 \\
& Speed of Engine & 0.536 \\
\hline
\end{tabular}

Figure 15 shows the battery SOC variation based on two control strategies under UDDS conditions. It can be seen that the battery SOC is stable at the end, and the changes are similar, indicating the effectiveness of the real-time EMS based on a BP neural network.

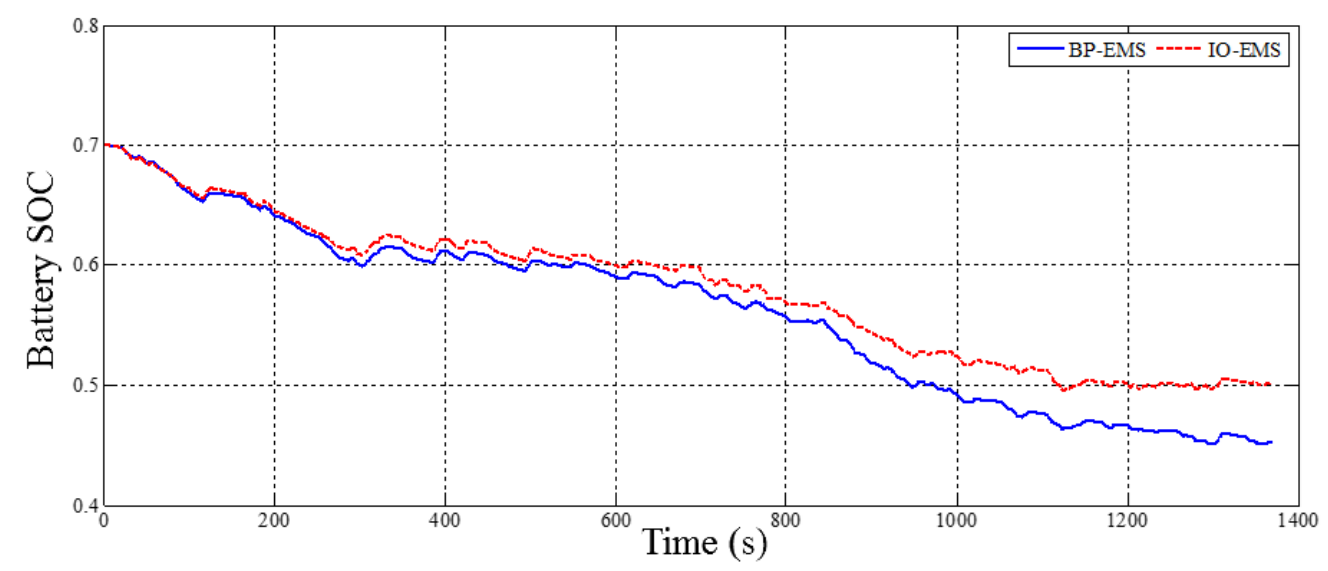

Figure 15. State of charge of battery during UDDS driving cycle. 
It can be seen from Table 4 that the BP-EMS can simulate the control rules of the IO-EMS well, and the control effect is similar to that of the IO-EMS. The average fuel consumption difference between these two strategies under various operating conditions is $1.2 \%$, while the average operation speed of the BP-EMS is improved effectively (the improvement degree is $98.1 \%$ ), which shows that the improved control strategy has a high application value.

Table 4. Fuel consumption and operation time in two strategies.

\begin{tabular}{ccccc}
\hline \multirow{2}{*}{ Cycle Condition } & \multicolumn{2}{c}{ BP-EMS } & \multicolumn{2}{c}{ IO-EMS } \\
\cline { 2 - 5 } & $\begin{array}{c}\text { Fuel Consumption } \\
\text { (L/100 } \mathbf{~ k m )}\end{array}$ & $\begin{array}{c}\text { Operation Time } \\
\mathbf{( s )}\end{array}$ & $\begin{array}{c}\text { Fuel Consumption } \\
\text { (L/100 km) }\end{array}$ & $\begin{array}{c}\text { Operation Time } \\
\text { (s) }\end{array}$ \\
\hline UDDS & 3.71 & 20 & 3.70 & 1230 \\
NEDC & 3.69 & 18 & 3.64 & 1059 \\
US06 HWY & 4.39 & 11 & 4.31 & 356 \\
\hline
\end{tabular}

\section{Conclusions}

In this paper, an instantaneous optimization strategy based on the principle of "minimum power loss" is developed for CSPM-HEV. To solve the problem of complex algorithms and poor real-time performance, in addition, acBP neural network is employed to realize the real-time energy management of CSPM-HEV. The results may be summarized as follows:

(1) The transmission ratio of the CSPM $i_{C S P M}$ was defined with reference to the traditional mechanical transmission, furthermore, the CSPM-HEV power distribution coefficient was raised for analyzing the system. Afterwards, the relationship about the power loss of the vehicle and $\left(i_{C S P M}, f_{1}\right)$ was derived, as a result, and the instantaneous optimization energy management based on the principle of "minimum power loss" is established. The strategy calculates the engine power and speed at the current time according to the optimal combination of $i_{C S P M}$ and $f_{1}$, so that achieve the instantaneous optimal control of the vehicle.

(2) According to the simulation results of the instantaneous optimization strategy under various working conditions, the learning samples were made. The vehicle power, speed and battery SOC were input variables, the engine power and speed were output variables, afterwards, the neural network controller was established, so the real-time energy management strategy based on a BP neural network is fulfilled.

(3) The real-time energy management strategy based on a BP neural network was simulated and compared with the results of a traditional instantaneous optimization strategy. The results show that the BP-EMS can greatly improve the running speed and optimize the control effect, and it also realizes the nonlinear mapping between engine output and drive axle demand power, speed and battery SOC, as a result, the instantaneous optimal control of CSPM-HEV is completed. In spite of benefits of BP-EMS, since the training sample of the BP neural network controller is the result of the instantaneous optimization strategy in the paper, it is unable to reach the global optimization. In the future, the authors will train samples based on the results of the global optimization algorithm, and employ a hybrid vehicle working condition recognition technology, which is expected to further improve the fuel economy of CSPM-HEV.

Author Contributions: Conceptualization, Q.X. and Y.M.; Methodology, Q.X.; Software, M.Z.; Validation, Q.X., Y.M. and M.Z.; Formal Analysis, Y.M.; Investigation, Y.M.; Resources, Q.X.; Data Curation, Q.X.; Writing-Original Draft Preparation, Q.X.; Writing-Review \& Editing, Q.X. and Y.M.; Supervision, Q.X., Project Administration, S.C.

Funding: This research was funded by (National Natural Science Foundation of China) grant number (51507021), Chongqing Science and Technology Commission of China under Project No. cstc2013jcyjA60001, graduate research and innovation foundation of Chongqing, China under Project No. CYS17008 and The State Key Laboratory of Power Transmission Equipment \& System Security and New Technology in Chongqing University of China under Project No. 2007DA10512716303.

Conflicts of Interest: The authors declare no conflict of interest. 


\section{References}

1. Hoeijmakers, M.J.; Ferreira, J.A. The electrical variable transmission. In Proceedings of the 39th IAS Annual Meeting, Seattle, WA, USA, 3-7 October 2004; Institute of Electrical and Electronics Engineers Inc.: Piscataway, NJ, USA, 2004.

2. Abdrakhmanov, R.; Adouane, L. Dynamic programming resolution and database knowledge for online predictive energy management of hybrid vehicles. In Proceedings of the 14th International Conference on Informatics in Control, Automation and Robotics (ICINCO 2017), Madrid, Spain, 26-28 July 2017; Volume 1, pp. 132-143.

3. Johannesson, L.; Asbogard, M.; Egardt, B. Assessing the potential of predictive control for hybrid vehicle powertrains using stochastic dynamic programming. IEEE Trans. Intell. Transp. Syst. 2007, 8, 71-83. [CrossRef]

4. Ouddah, N.; Adouane, L.; Abdrakhamanov, R.; Kamal, E. Optimal energy management strategy of plug-in hybrid electric bus in urban conditions. In Proceedings of the 14th International Conference on Informatics in Control, Automation and Robotics (ICINCO 2017), Madrid, Spain, 26-28 July 2017; Volume 1, pp. 304-311.

5. Amini, M.H.; Karabasoglu, O. Optimal operation of interdependent power systems and electrified transportation networks. Energies 2018, 11, 196. [CrossRef]

6. Qi, X.; Wu, G.; Boriboonsomsin, K.; Barth, M.J. An on-line energy management strategy for plug-in hybrid electric vehicles using an estimation distribution algorithm. In Proceedings of the 17th International IEEE Conference on Intelligent Transportation Systems (ITSC), Qingdao, China, 8-11 October 2014; pp. $2480-2485$.

7. Qi, X.; Wu, G.; Boriboonsomsin, K.; Barth, M.J. Development and evaluation of an evolutionary algorithm-based online energy management system for plug-in hybrid electric vehicles. IEEE Trans. Intell. Transp. Syst. 2017, 18, 2181-2191. [CrossRef]

8. Peng, J.; Fan, H.; He, H.; Pan, D. A rule-based energy management strategy for a plug-in hybrid school bus based on a controller area network bus. Energies 2015, 8, 5122-5142. [CrossRef]

9. Hofman, T.; van Druten, R.M.; Serrarens, A.F.A.; Steinbuch, M. Rule-based energy management strategies for hybrid vehicles. Int. J. Elect. Hybrid Veh. 2007, 1, 71-94. [CrossRef]

10. Hofman, T.; Steinbuch, M.; van Druten, R.M.; Serrarens, A.F.A. Rule-based energy management strategies for hybrid vehicle drivetrains: A fundamental approach in reducing computation time. In Proceedings of the 4th IFAC Symposium on Mechatronic Systems, Heidelberg, Germany, 12-14 September 2006; pp. 1-6.

11. Adel, B.; Youtong, Z.; Shua, S. Parallel HEV hybrid controller modeling for power management. World Electr. Veh. J. 2010, 4, 190-196. [CrossRef]

12. Hajizadeh, A.; Golkar, M.A. Intelligent power management strategy of hybrid distributed generation system. Electr. Power Energy Syst. 2007, 29, 783-795. [CrossRef]

13. Lihao, Y.; Youjun, W.; Congmin, Z. Study on fuzzy energy management strategy of parallel hybrid vehicle based on quantum PSO algorithm. Int. J. Multimedia Ubiquitous Eng. 2016, 11, 147-158. [CrossRef]

14. Denis, N.; Dubois, M.R.; Desrochers, A. Fuzzy-based blended control for the energy management of a parallel plug-in hybrid electric vehicle. Intell. Transp. Syst. 2015, 9, 30-37. [CrossRef]

15. Martnez, C.M.; Hu, X.; Cao, D.; Velenis, E.; Gao, B.; Weller, M. Energy management in plug-in hybrid electric vehicles: Recent progress and a connected vehicles perspective. IEEE Trans. Veh. Technol. 2017, 66, 4534-4549. [CrossRef]

16. Dai, X.; Li, C.K.; Rad, A.B. An approach to tune fuzzy controllers based on reinforcement learning for autonomous vehicle control. IEEE Trans. Intell. Transp. Syst. 2005, 6, 285-293. [CrossRef]

17. Qi, X.; Wu, G.; Boriboonsomsin, K.; Barth, M.J.; Gonder, J. Data driven reinforcement learning-based real-time energy management system for plug-in hybrid electric vehicles. Transp. Res. Rec. J. Transp. Res. Board 2016, 2572, 1-8. [CrossRef]

18. Qi, X.; Luo, Y.; Wu, G.; Boriboonsomsin, K.; Barth, M.J. Deep reinforcement learning-based vehicle energy efficiency autonomous learning system. In Proceedings of the 2017 IEEE Intelligent Vehicles Symposium (IV), Los Angeles, CA, USA, 11-14 June 2017; pp. 1228-1233.

19. Zhang, X.; Liu, Y.; Zhang, J. A Fuzzy Neural Network Energy Management Strategy for Parallel Hybrid Electric Vehicle. In Proceedings of the 9th International Conference on Modelling, Identification and Control (ICMIC 2017), Kunming, China, 10-12 July 2017. 
20. Xu, Q.; Cui, S.; Song, L.; Zhang, Q. Research on the Power Management Strategy of Hybrid Electric Vehicles Based on Electric Variable Transmissions. Energies 2014, 7, 934-960. [CrossRef]

21. Xu, Q.; Sun, J.; Luo, L.; Cui, S.; Zhang, Q. A Study on Magnetic Decoupling of Compound-Structure Permanent-Magnet Motor for HEVs Application. Energies 2016, 9, 819. [CrossRef]

22. Wang, X. Introduction of Neural Networks; Science Press of China: Beijing, China, 2017. 